\title{
Structural Effects of Cation Binding to DPPC Monolayers
}

\author{
Matti Javanainen, ${ }^{*}, \dagger$ Wei Hua ${ }^{\ddagger}$ Ondřej Ticháček, ${ }^{\dagger}$ Pauline Delcroix, $₫$ Lukasz \\ Cwiklik, and Heather C. Allen*,‡ \\ $\dagger$ Institute of Organic Chemistry and Biochemistry of the Czech Academy of Sciences, \\ Flemingovo nám. 542/2, 16000 Prague 6, Czech Republic \\ $\ddagger$ Department of Chemistry and Biochemistry, The Ohio State University, Columbus, Ohio \\ 43210, United States \\ 9J. Heyrovsky Institute of Physical Chemistry of the Czech Academy of Sciences, \\ Dolejskova 3, 18223 Prague 8, Czech Republic \\ E-mail: matti.javanainen@gmail.com; allen@chemistry.ohio-state.edu
}

\begin{abstract}
Ions at the two sides of the plasma membrane maintain the transmembrane potential, participate in signaling, and affect the properties of the membrane itself. The extracellular leaflet is particularly enriched in phosphatidylcholine lipids an under the influence of $\mathrm{Na}^{+}, \mathrm{Ca}^{2+}$, and $\mathrm{Cl}^{-}$ions. In this work, we combined molecular dynamics simulations performed using state-of-the-art models with vibrational sum frequency generation (VSFG) spectroscopy to study the effects of these key ions on the structure of dipalmitoylphosphatidylcholine. We used lipid monolayers as a proxy for membranes, as this approach enabled a direct comparison between simulation and experiment. We find that the effects of $\mathrm{Na}^{+}$are minor. $\mathrm{Ca}^{2+}$, on the other hand, strongly affects the lipid head group conformations and induces a tighter packing of lipids, thus promoting
\end{abstract}


the liquid condensed phase. It does so by binding to both the phosphate and carbonyl oxygens via direct and water-mediated binding modes, the ratios of which depend on the monolayer packing. Clustering analysis performed on simulation data revealed that changes in area per lipid or $\mathrm{CaCl}_{2}$ concentration both affect the head group conformations, yet their effects are anti-correlated. Cations at the monolayer surface also attract $\mathrm{Cl}^{-}$, which at large $\mathrm{CaCl}_{2}$ concentrations penetrates deep to the monolayer. This phenomenon coincides with a radical change in the VSFG spectra of the phosphate group, thus indicating the emergence of a new binding mode.

\section{Introduction}

Concentration gradients of cations across cellular membranes are required to maintain the electrostatic potentials across these membranes. This potential can be harnessed to drive transmembrane processes, and its localized changes are used as a means of signaling. Moreover, cations such as calcium also directly participate in numerous signaling pathways at the membrane surface. ${ }^{1-3}$ Cation adsorption can also affect the lateral structure of lipid membranes and vice versa ${ }^{4-6}$ which could regulate membrane protein function via membranemediated effects. Therefore, understanding the interactions between cations and lipid membranes is of crucial interest - a task best tackled with a collaboration between simulations and experiments.

Indeed, numerous molecular dynamics (MD) simulation studies have focused on lipid-ion interactions in an attempt to reveal atom-level information of these interactions. ${ }^{6-10}$ Unfortunately, it has only recently become evident that in most of these studies the adsorption of both sodium and calcium onto phosphatidylcholine (PC) head groups has been significantly overestimated. ${ }^{8}$ While lipid bilayer simulations are routinely performed in silico, they are often not convenient to study experimentally. Therefore, lipid monolayers are often used as a model of the bilayer. ${ }^{11}$ In contrast, MD simulations have traditionally struggled with lipid monolayers ${ }^{12}$ due to the inadequate description o6 water-air interfaces. These compatibil- 
ity issues between experiment and simulation have traditionally prevented quantitative and interdisciplinary studies on lipid-ion interactions.

In this work, to overcome the aforementioned issues, we complemented experimental work on lipid monolayers with simulations that bring together three key methodological improvements in the field. Namely, we combined a recent ECC lipid model by Melcr et al., ${ }^{13}$ the ECC ions, ${ }^{14-16}$ and the optimal point charge (OPC) water model. The ECC ("electronic continuum correction") ions account for electronic polarizability in a mean-field manner by using scaled-down charges. The ECC lipid model is based on the Lipid14 ${ }^{17}$ model and correctly describes the adsorption of ECC ions onto the PC head group region. ${ }^{13}$ Finally, the OPC water model ${ }^{18}$ was recently shown to be able to describe lipid monolayer behavior, ${ }^{19,20}$ and thus avoid many of the issues with MD simulations of such systems. ${ }^{12}$

With this state-of-the-art set of force fields and in combination with Langmuir trough measurements and vibrational sum frequency generation (VSFG) spectroscopy, we studied the effect of the key cations $-\mathrm{Na}^{+}$and $\mathrm{Ca}^{2+}$ - on the properties of DPPC monolayers. While such monolayes serve as models for lipid membranes, they are also interesting in the context of the DPPC-rich pulmonary surfactant. ${ }^{21,22}$

We found that $\mathrm{Na}^{+}$has little effect on the monolayer properties. However, $\mathrm{Ca}^{2+}$ causes a significant decrease in the surface pressure of the monolayer by favoring the presence of the liquid condensed phase. We also found that a few hundred millimolar concentrations of $\mathrm{CaCl}_{2}$ are able to induce this effect, whereas larger concentrations do not have a substantial effect on the surface pressure. Nevertheless, at these larger concentrations, lipid head group conformations are still affected as both $\mathrm{Ca}^{2+}$ and $\mathrm{Cl}^{-}$are able to penetrate and bind deep into the lipid carbonyl region and displace hydration water. This observation is supported by the VSFG spectra of phosphate stretching, which also indicates an emergence of a specific $\mathrm{Ca}^{2+}-\mathrm{PO}_{4}^{2-}$ complex. 


\section{Methods}

\section{Simulation Models and Methods}

Atomistic classical molecular dynamics simulations were performed to unravel the effect of cations on the structure of a dipalmitoylphosphatidylcholine (DPPC) monolayer. The simulated systems contained 512 DPPC molecules in two independent monolayers, each with 256 lipids. These lipid films were located at the water-air interfaces formed on a slab of $\sim 40,000$ water molecules located in the middle of a periodic simulation box. Monolayers with

eight area per lipid (APL) values $\left(42,48,54,60,66,72,78,90 \AA^{2}\right)$ were simulated. Hence, the simulated systems captured the liquid condensed $\left(\mathrm{L}_{\mathrm{c}}\right)$ and liquid expanded $\mathrm{L}_{\mathrm{e}}$ phases as well as their coexistence. The lateral sizes of the simulation box ranged from approximately $10 \times 10 \mathrm{~nm}^{2}$ to $15 \times 15 \mathrm{~nm}^{2}$. The box was elongated to $22 \mathrm{~nm}$ in the direction normal to the interface in order to avoid long-range electrostatic interactions of lipids through the vacuum (air) phase. Snapshots of the monolayer at three different APL values are shown in Fig. 1.

In terms of solvent, either pure water or solutions of $\mathrm{NaCl}$ or $\mathrm{CaCl}_{2}$ were considered. For $\mathrm{NaCl}, 600 \mathrm{Na}^{+}$and $\mathrm{Cl}^{-}$ions were inserted, which yielded a concentration of $\sim 820 \mathrm{mM}$. For $\mathrm{CaCl}_{2}, 300 \mathrm{Ca}^{2+}$ ions were included with twice the amount of $\mathrm{Cl}^{-}$counterions to provide a concentration of $410 \mathrm{mM}$. Additionally, monolayers with APLs equal to 48, 66, and $78 \AA^{2}$, corresponding to the $\mathrm{L}_{c}$ phase, $\mathrm{L}_{\mathrm{c}} / \mathrm{L}_{\mathrm{e}}$ coexistence, and the $\mathrm{L}_{\mathrm{e}}$ phase, were simulated with different amounts of $\mathrm{CaCl}_{2}$. To this end, 75, 150, 300, 750, or $1500 \mathrm{Ca}^{2+}$ ions were included with twice the amount of $\mathrm{Cl}^{-}$counterions to provide concentrations of $101 \mathrm{mM}, 203 \mathrm{mM}$, $1.06 \mathrm{M}$, and $2.25 \mathrm{M}$ (note that ions are added by replacing water molecules with them).

In the case of both pure water and $\mathrm{NaCl}, 200$ ns-long trajectories were simulated whereas 300 ns-long trajectories were collected for the systems containing $\mathrm{CaCl}_{2}$. In each case, the last 100 ns were used for analyses. Simulations were performed employing canonical ensemble at a temperature of $298 \mathrm{~K}$. The simulation outputs are available at doi.org/10.5281/ zenodo.1043958 (no ions), doi.org/10.5281/zenodo.1043956 (NaCl), and doi.org/10. 

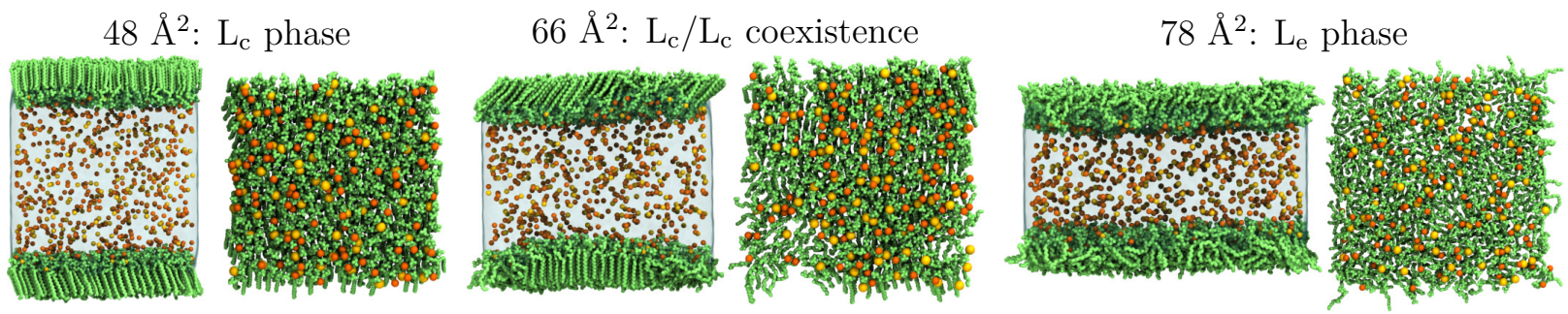

Figure 1: Side and top views of selected systems with $410 \mathrm{mM} \mathrm{CaCl}_{2}$ and at varying APLs. These correspond to the $\mathrm{L}_{\mathrm{c}}$ phase $\left(48 \AA^{2}\right)$, the $\mathrm{L}_{\mathrm{e}}$ phase $\left(78 \AA^{2}\right)$, and their coexistence $\left(66 \AA^{2}\right)$. DPPC is shown in green, $\mathrm{Ca}^{2+}$ in yellow, $\mathrm{Cl}^{-}$in orange, and water as a transparent blue surface. Hydrogens are omitted for clarity.

To accurately capture both the lipid behavior at a water-air interface and the cation affinity for the PC head group, we combined a set of state-of-the-art force fields. Lipids were modeled using the headgroup parameters adapted from ECC-POPC, ${ }^{13}$ which is based on Lipid14. ${ }^{17}$ This lipid model was parameterized to capture the head group responses to cations when combined with the ion models developed by the group of Pavel Jungwirth. ${ }^{14-16}$ Briefly, these ion models account for electronic polarizability in a mean field manner by adapting the electronic continuum correlation (ECC) approach. ${ }^{23-26}$ Effectively, the charges of the ions are scaled by a factor of 0.75 and the Lennard-Jones $\sigma$ parameter is fine-tuned to agree with neutron scattering data. Finally, the OPC water model ${ }^{18}$ was recently demonstrated by us to provide good agreement with experimental isotherms of lipid monolayers ${ }^{19,20}$ and was hence adapted to this work. Since the ECC ions were parametrized with another water model $(\mathrm{SPC} / \mathrm{E})$, we refined their $\sigma$ parameters so that the first peak in the water-ion radial distribution function agreed with experimental findings (see Table S1 in the SI).

The simulations were performed using GROMACS v5.1.4. ${ }^{27}$ Periodic boundary conditions were used in all directions and the Newtonian equations of motion were integrated with a time step of 2 fs. Lennard-Jones potential was shifted to zero at $1.2 \mathrm{~nm}$, and the smooth particle mesh Ewald method ${ }^{28}$ was used to calculate the long-range contribution to electrostatic interactions. The dispersion correction ${ }^{29}$ was applied to energy and pressure to account for 
long-range Lennard-Jones interactions. Neighbor lists were maintained using buffered Verlet lists. ${ }^{30}$ The temperatures of the lipids and the solvent were separately coupled to a stochastic velocity rescaling thermostat ${ }^{31}$ with a target temperature of $298 \mathrm{~K}$ and a time constant of 1 ps. The bonds involving hydrogens were constrained using the LINCS algorithm. ${ }^{32,33}$

\section{Simulation Analyses}

The surface pressures $\Pi$ at an APL of $A$ were calculated from the surface tensions $\gamma$ measured from simulations as

$$
\Pi(A)=\gamma_{0}-\gamma(A),
$$

where $\gamma_{0}$ is the surface tension of the lipid-free solvent-air interface, and due to the symmetry of the simulation system, $\gamma$ is calculated simply as

$$
\gamma=\frac{\left(P_{\mathrm{N}}-P_{\mathrm{L}}\right) \times L_{z}}{2},
$$

where $P_{\mathrm{N}}$ and $P_{\mathrm{L}}=1 / 2 \times\left(P_{x x}+P_{y y}\right)$ are the lateral and normal components of the pressure, $P_{x x}=P_{y y}$ are the lateral components of the pressure tensor, and $L_{z}$ is the length of the simulation box normal to the interface. The value of $\gamma_{0}$ is sensitive to ions so its values were extracted for all used ionic conditions from simulations without lipids.

The fractions of the $\mathrm{L}_{e}$ phase were calculated as in our previous work. ${ }^{19}$ The oxygencation contacts were measured with a cutoff of $0.325 \mathrm{~nm}$ as in our earlier work. ${ }^{6}$ For chloride, we set the cut-off to $0.535 \mathrm{~nm}$ as it includes the first peak in the oxygen-chloride RDF. Density profiles were calculated with the gmx density tool bundled with GROMACS. The profiles were centered based on the lipid coordinates, and symmetrized. The numbers of $\mathrm{Ca}^{2+}$ and $\mathrm{Cl}^{-}$ions that penetrated below the phosphate region was calculated as follows:

The number densities of the ions were integrated from the maximum of the phosphate density until the vacuum phase. This maximum density was obtained as the midpoint of a Gaussian 
fit to the phosphate density profile.

The relative numbers of ions bound directly and in a water-mediated manner to lipids were calculated as follows: non-normalized radial distribution functions (RDFs) of ions around carbonyl and phosphorus (the two bound only to phosphorus) oxygens were calculated. The first peak (direct binding) was numerically integrated from 0 to $0.32 \mathrm{~nm}$, which lies in the first wide minimum. For carbonyl, the top of the second peak was fitted with a Gaussian function and analytically integrated, whereas for phosphate a double Gaussian was used to capture the clearly visible bimodal distribution. These Gaussians were used as the RDF did not return to zero after the second peak and hence we wanted to avoid setting an arbitrary cutoff distance. The coordination numbers were estimated by integrating RDFs of carbonyl and phosphate (the two bound only to phosphorus) oxygens up to the minimum after the second peak.

Conformations of the lipids were analysed statistically by a computer clustering method. Data from all simulations were combined and the pairwise inter-lipid distances were calculated. The distance was defined as the Euclidean distance between the positions of the head-group atoms which were first aligned. The alignment (translation and rotation) was done in a pairwise fashion using a rigid part of glycerol backbone, namely the $\mathrm{C} 1, \mathrm{C} 2$ and C3 atoms. Hierarchical clustering method using the Ward's linkage method was applied on a total of 359424 lipid conformations randomly sampled from 18 individual simulations with varying conditions (area per lipid of 48,66 , or $78 \AA^{2}$ and $\mathrm{CaCl}_{2}$ concentration of 0 , $101,203,410,1060$, or $2250 \mathrm{mM})$. The trajectory was pre-processed with the help of the MDAnalysis python package. ${ }^{34}$ The pairwise distance was calculated using an in-house developed software employing an optimized singular value decomposition method based on Ref. 35 and implemented by Eric V. Jang (https://github.com/ericjang/svd3). The clustering was carried out using a modified version of the scipy.cluster.hierarchy Python package https://github.com/scipy/scipy/tree/v1.5.2/scipy/cluster.

Deuterium order parameter is defined as $S_{\mathrm{CD}}=\frac{1}{2}\left\langle 3 \cos ^{2} \theta-1\right\rangle$. The $S_{\mathrm{CD}}$ values for the 
head group $\beta, \alpha$ and $g_{1}$ carbons (see Fig. 10) were calculated from the carbon and hydrogen positions with an in-house script. The values for the two hydrogens were calculated and the mean and average of these two values were used as the value and error for each studied carbon.

Visualization was performed using the VMD Molecular Graphics Viewer ${ }^{36}$ and rendered with the use of the Tachyon Ray Tracing System. ${ }^{37}$

\section{Materials}

Sodium chloride $(\mathrm{NaCl})$ (certified ACS, 99.5-100.5\%) and calcium chloride hexahydrate $\left(\mathrm{CaCl} 2 \cdot 6 \mathrm{H}_{2} \mathrm{O}\right)$ (certified ACS, 99\%) were purchased from Fisher Scientific (Waltham, MA). 1,2-dipalmitoyl-sn-glycero-3-phosphocholine (DPPC) (>99\%, Avanti Polar Lipids, Alabaster, AL) was used as received and dissolved in chloroform (>99.9\%, HPLC grade, Sigma-Aldrich, St. Louis, MO) at a $1 \mathrm{mM}$ concentration. Ultrapure water with a resistivity of $18.2-18.3$ $\mathrm{M} \Omega \cdot \mathrm{cm}$ and measured $\mathrm{pH}$ of 5.6 (the $\mathrm{pH}$ is slightly acidic due to the dissolution of gaseous $\mathrm{CO}_{2}$ ) was obtained from a Barnstead Nanopure system (D4741, Thermolyne Corporation, Dubuque, IA) equipped with additional organic removing cartridges (D5026 Type I ORGANICfree Cartridge Kit; Pretreat Feed). Stock chloride salt solutions were prepared by dissolving salts in ultrapure water and pretreated according to procedures described previously to remove potential remaining organic contamination. ${ }^{38}$ Concentrations of these solutions were standardized by Mohr titration. ${ }^{39}$ Once the concentrations were known, each salt stock solution was diluted to the desired concentrations. The measured $\mathrm{pH}$ of the salt solutions laid in the range 5-7. All salt solutions were shown to be free of organic impurities, as revealed by their VSFG spectra obtained in the surfactant $\mathrm{CH}$ stretching region $\left(2800-3000 \mathrm{~cm}^{-1}\right)$. All solutions were thermally equilibrated to room temperature $\left(23 \pm 1^{\circ} \mathrm{C}\right)$ over $24 \mathrm{~h}$ prior to measurement. 


\section{VSFG Spectroscopy Measurements}

VSFG spectra $\left(\left|\chi_{\mathrm{S}}^{(2)}\left(\omega_{\mathrm{IR}}\right)\right|^{2}\right)$ from the lipid-covered aqueous surfaces were obtained on a broad-bandwidth VSFG spectrometer setup that has been described elsewhere. ${ }^{40}$ The average visible/infrared (IR) pulse energy of the visible $(0.8 \mu \mathrm{m})$ and IR $(2.6-3.3 \mu \mathrm{m}$ and 8.3-10.0 $\mathrm{mm}$ in the water $\mathrm{OH}$ and phosphate stretching regions, respectively) beams incident on the aqueous surface were $300 / 6$ and $300 / 10 \mu \mathrm{J}$ for the phosphate and water $\mathrm{OH}$ spectral regions, respectively. Spectra were recorded with a 5 min integration time. All VSFG spectra were background-subtracted and normalized against the non-resonant VSFG spectrum from a GaAs(110) crystal (Lambda Research Optics, Costa Mesa, CA) to eliminate the spectral distortion caused by the frequency-dependent IR beam energy in the spectral region of interest. For each experiment, ultrapure water was poured ( $28 \mathrm{~mL})$ in acid-cleaned Petri dishes (5 cm diameter) and spectra of the neat air/water interface were used as a reference to assess reproducibility during the entire experimental period. Then, water was replaced by aqueous salt solutions onto which DPPC monolayers were overspread $(\sim 12 \mu \mathrm{L})$. Under this condition, DPPC monolayers were equilibrated in the highly ordered liquid-condensed (LC) phase as tested by surface tension measurement. Prior to any spectroscopic measurements, 10 min was allowed for solvent evaporation. The $\operatorname{ssp}$ ( $s$ for sum-frequency, $s$ for visible, and $p$ for infrared) polarization combination was selected for all spectra presented here. All measurements were repeated at least three times to ensure reproducibility and were recorded at room temperature $\left(23 \pm 1^{\circ} \mathrm{C}\right)$. Only every second data points are plotted in the VSFG spectra to avoid spectral clutter. 


\section{Results}

\section{$\mathrm{Ca}^{2+}$ but not $\mathrm{Na}^{+}$Induces a Shift In the Pressure-Area Isotherm}

We first calculated the surface pressure-area isotherms for DPPC in the presence and absence of ions and compared them against experimentally measured isotherms for similar systems. ${ }^{41}$ In simulations, the surface tension of the pure water-air interface was calculated to be $70.9 \pm 0.3 \mathrm{mN} / \mathrm{m}$, in good agreement with the experimental value of $71.97 \mathrm{mN} / \mathrm{m} .{ }^{42}$ The ions had little effect on this tension, yet the $\gamma_{0}$ value in Eq. (1) was still calculated separately for each ion concentration. As the surface tension values of electrolyte solutions in Fig. S1 in the Supporting Information (SI) demonstrate, the used ECC ions ${ }^{14-16}$ indeed underestimate the effect of increasing salt concentration. This results from the fact that the charges should in principle be scaled by different factors at the two sides of the interface, which is also manifested by the fact that full charges tend to overestimate the dependence of surface tension on salt concentration. ${ }^{26}$

Both experimental and computational isotherms are shown in Fig. 2. The computational ones were calculated from the last $100 \mathrm{~ns}$ of simulation data so that the number of bound ions at the surface had equilibrated (see top row of Fig. S2 in the SI). The experimental isotherms (top panel, adapted from Ref. 41) show that $\mathrm{NaCl}$ has little effect on the shape and position of the isotherm, whereas $\mathrm{CaCl}_{2}$ shifts the isotherm downwards in the coexistence region. This behavior is also captured well in the computational isotherms (middle panel), where $\mathrm{NaCl}$ has little effect on the isotherms and $\mathrm{CaCl}_{2}$ mainly affects the pressure at the coexistence plateau. The numbers of cation-lipid interactions, plotted in the bottom panel of Fig. 2, reveal that $\mathrm{Na}^{+}$binds little to the lipids regardless of the monolayer APL. $\mathrm{Ca}^{2+}$, on the other hand, binds much more to lipids, and the amount of bound cations depends on the APL. Between 42 and $60 \AA^{2}$, the cation-lipid contacts increase by $\sim 40 \%$. Further increase in APL does not increase the number of these contacts.

We also studied the interaction of the chloride ions with the lipids. A longer cutoff was 

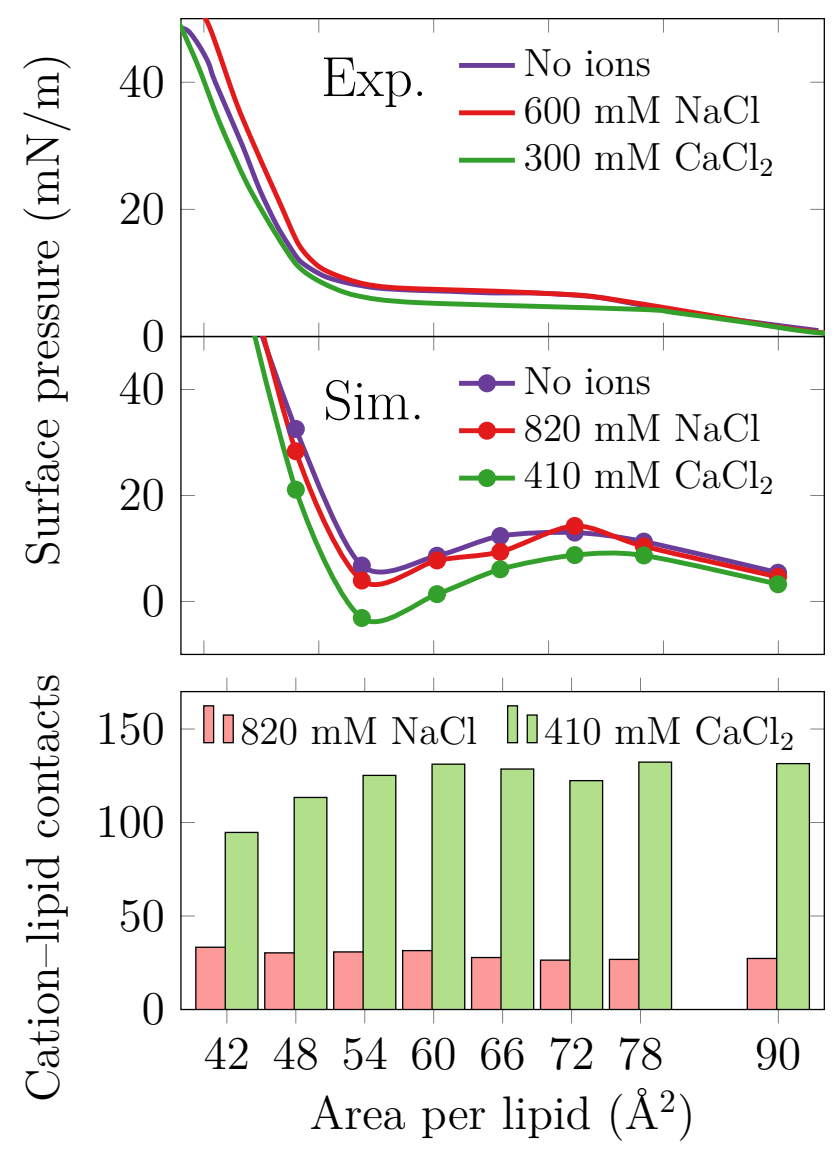

Figure 2: Experimental (top panel, adapted from Ref. 41) and simulated (middle panel) isotherms of the DPPC monolayer under various solvent environments. Note that the used concentrations between experiments and simulations are not equal. Still, in both cases $\mathrm{NaCl}$ has no effect on the isotherm, whereas $\mathrm{CaCl}_{2}$ induces a downward shift especially in the $\mathrm{L}_{\mathrm{c}} / \mathrm{L}_{\mathrm{e}}$ coexistence region. The number of cation-lipid contacts is shown in the bottom panel. For $\mathrm{NaCl}$, this number is small and independent of APL, whereas for $\mathrm{CaCl}_{2}$ the number of contacts increases upon increasing APL, until it saturates at an APL of $\sim 60 \AA^{2}$.

used to analyze the lipid-ion contacts (see Methods) since $\mathrm{Cl}^{-}$ions do not bind to the lipids directly, but instead are bridged to negative lipid groups via cations. As demonstrated in the bottom row in Fig. S2 in the SI, we observed that in the case of $\mathrm{NaCl}$, the larger the APL, the more chlorides were located in the vicinity of the lipids. This is somewhat surprising considering that the number of bound $\mathrm{Na}^{+}$ions is constant across the studied APLs. For $\mathrm{CaCl}_{2}$, the $\mathrm{Cl}^{-}$binding over time follows a similar pattern as that of $\mathrm{Ca}^{2+}$, and a similar dependency on APL is observed. 


\section{$\mathrm{Ca}^{2+}$ Bridges Lipid Head Groups and Thereby Promotes the $\mathbf{L}_{\mathrm{c}}$ Phase}

We next studied how the ions affect the packing of lipids at a nanoscopic level within the coexistence region. To this end, we calculated the fraction of lipid chains that were looselypacked and hence in the liquid expanded $\left(\mathrm{L}_{\mathrm{e}}\right)$ phase. The analysis was adopted from our previous work, ${ }^{19}$ and the results are shown in Fig. 3. At $54 \AA^{2}$, the entire monolayer is in the liquid condensed $\left(\mathrm{L}_{c}\right)$ phase, whereas the fraction of the $\mathrm{L}_{\mathrm{e}}$ phase grows steadily for lipid areas between 60 and $72 \AA^{2}$. The growth is similar independent of the presence of ions, yet the values for the system with $\mathrm{CaCl}_{2}$ are always somewhat lower than for the ionfree monolayer and the monolayer with $\mathrm{NaCl}$. This indicates that $\mathrm{CaCl}_{2}$ can induce tighter packing of the lipids, likely by clustering the head groups of multiple lipid molecules.

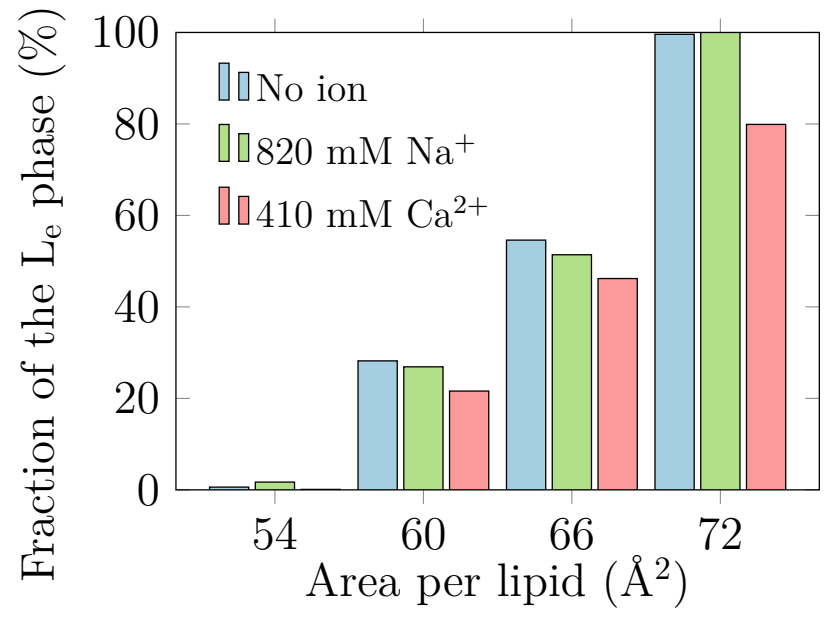

Figure 3: The fraction of the $\mathrm{L}_{\mathrm{e}}$ phase of the DPPC monolayer as a function of solvent environment in systems that are within the $\mathrm{L}_{\mathrm{c}} / \mathrm{L}_{\mathrm{e}}$ coexistence plateau.

A detailed look into the direct binding modes of $\mathrm{Ca}^{2+}$ to lipid oxygens is provided in Fig. S4 in the SI. In the radial distribution functions (RDFs), this direct binding has a sharp distribution at $0.21-0.23 \mathrm{~nm}$ (data not shown). Upon growing area, the repulsion between cations decreases and thus more $\mathrm{Ca}^{2+}$ binds to the phosphate groups. In the carbonyl region the situation is different as the number of $\mathrm{Ca}^{2+}$ penetrating there is also much smaller. At the smallest area, less $\mathrm{Ca}^{2+}$ reaches the carbonyl region likely due to the compact packing, yet 
at larger areas the number of $\mathrm{Ca}^{2+}$ bound to carbonyls is fairly constant. Only at the largest area per lipid, the number of bound $\mathrm{Ca}^{2+}$ slightly decreases, likely due to the dynamic nature of the $\mathrm{L}_{\mathrm{e}}$ phase monolayer which makes it difficult for the cations to engage in optimally coordinated binding modes.

In addition to these direct binding modes, we also analyzed the solvent-shared $\mathrm{Ca}^{2+}-$ lipid binding. For carbonyl oxygens, the number of $\mathrm{Ca}^{2+} \mathrm{s}$ bound via water molecules was $\sim 2.2$ times that of the directly-bound ones and the RDFs had a single Gaussian distribution centered at $\sim 0.46 \mathrm{~nm}$.

For the phosphates, the situation is quite different: The solvent-shared binding displayed a bimodal distribution with characteristic $\mathrm{Ca}^{2+}$-oxygen distances of $0.39 \mathrm{~nm}$ and $0.44 \mathrm{~nm}$. These corresponds to modes where the binding is coordinated by two or one water molecules, respectively. For the former, the $\mathrm{Ca}^{2+}$, oxygen, and the two waters form a rhombus-like shape and thus the $\mathrm{Ca}^{2+}$-oxygen distance is shorter than in the case of a single water bridging the $\mathrm{Ca}^{2+}$ and oxygen in a linear arrangement. The conformation with a single shared water molecule was always more prevalent by $\sim 50 \%$. However, unlike for the carbonyls where the solvate-shared binding dominates, the number of $\mathrm{Ca}^{2+}$ bound by solvent-sharing to phosphate oxygens was $10 \%$ lower than the number of directly bound $\mathrm{Ca}^{2+}$.

The coordination number of carbonyl and phosphate oxygens around $\mathrm{Ca}^{2+}$ at $0.32 \mathrm{~nm}$ ranged from 1.0 to 1.4, growing monotonously with area per lipid. However, a molecule can reside close to both carbonyl and phosphate oxygen of the same lipid, so our numbers are likely somewhat overestimated. At the end of the second peak in the $\mathrm{Ca}^{2+}$-oxygen $\mathrm{RDF}$ at $0.53 \mathrm{~nm}$, the coordination numbers ranged from 2.2 to 2.9 . These numbers indicate that $\mathrm{Ca}^{2+}$ directly binds to one lipid, yet it can bind to another 1-2 lipids via solvent-shared binding. 


\section{Higher $\mathrm{CaCl}_{2}$ Concentrations Affect Lipid Head Group Conforma- tion Instead of Lipid Packing}

Next, we studied the effect of $\mathrm{CaCl}_{2}$ concentration on the monolayer at selected values for the area per lipid. These systems corresponded to the $\mathrm{L}_{\mathrm{e}}$ and $\mathrm{L}_{\mathrm{c}}$ phases as well their coexistence, and the studied $\mathrm{CaCl}_{2}$ concentrations were 101, 203, 410, 820, 1060, and $2250 \mathrm{mM}$. We again verified that the number of bound ions had stabilized before the last $100 \mathrm{~ns}$ of the simulation trajectory that was used in the analyses (see top row Fig. S3 in the SI). We first calculated the dependency of the surface pressure on the $\mathrm{CaCl}_{2}$ concentration, which is shown in Fig. 4. It is clear that at small $\mathrm{CaCl}_{2}$ concentrations, the surface pressure values drop, yet the curves stabilize at around $400 \mathrm{mM}$. This could either mean that the monolayer is saturated with ions at that concentration, or that the additional adsorption of ions has no effect on the packing of the monolayer. Further analysis demonstrates that the effect of $\mathrm{CaCl}_{2}$ concentration on the $\mathrm{L}_{\mathrm{e}}$ fraction was also negligible at concentrations larger than $400 \mathrm{mM}$ (see the inset of Fig. 4.

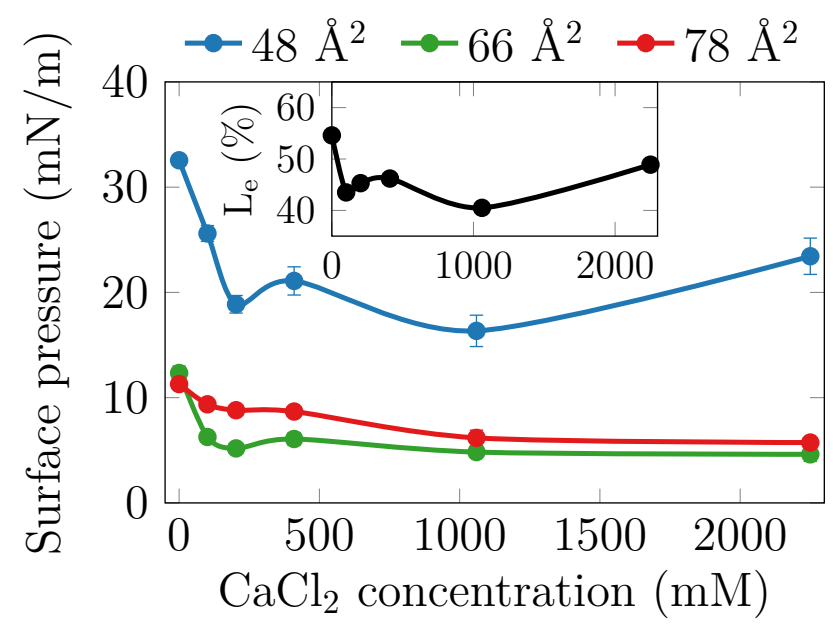

Figure 4: The surface pressures of the simulated DPPC monolayers in different phases as a function of $\mathrm{CaCl}_{2}$ concentration. The inset shows the fraction of the $\mathrm{L}_{\mathrm{e}}$ phase as a function of $\mathrm{CaCl}_{2}$ concentration for the monolayer with an APL of $66 \AA^{2}$.

The number of contacts between lipid oxygens and calcium ions, shown in Fig. 5, reveal 
that no such saturation of ion adsorption is observed in the studied concentration range. This is also highlighted by the time evolution of the lipid- $\mathrm{Ca}^{2+}$ contacts shown on the top row of Fig. S3 in the SI. At small $\mathrm{CaCl}_{2}$ concentrations, the binding to monolayers with different APLs and hence different phases is fairly equal, whereas at higher concentrations the adsorption to monolayers with larger APL values is substantially larger. At high $\mathrm{CaCl}_{2}$ concentrations, the binding to the carbonyl groups seems to be somewhat saturated and there is little difference between binding to the these groups in the monolayers with the two largest APLs. However, at the high $\mathrm{CaCl}_{2}$ concentrations, the phosphate oxygens bind a substantial amount of cations. As discussed earlier, this binding to phosphates seems to have little effect on lipid packing and hence on surface pressure (see Fig. 4). The binding of $\mathrm{Cl}^{-}$ions to lipids via $\mathrm{Ca}^{2+}$ bridges again showed the same shape and tendency as the $\mathrm{Ca}^{2+}$ binding (see bottom row in Fig. S3 in the SI).

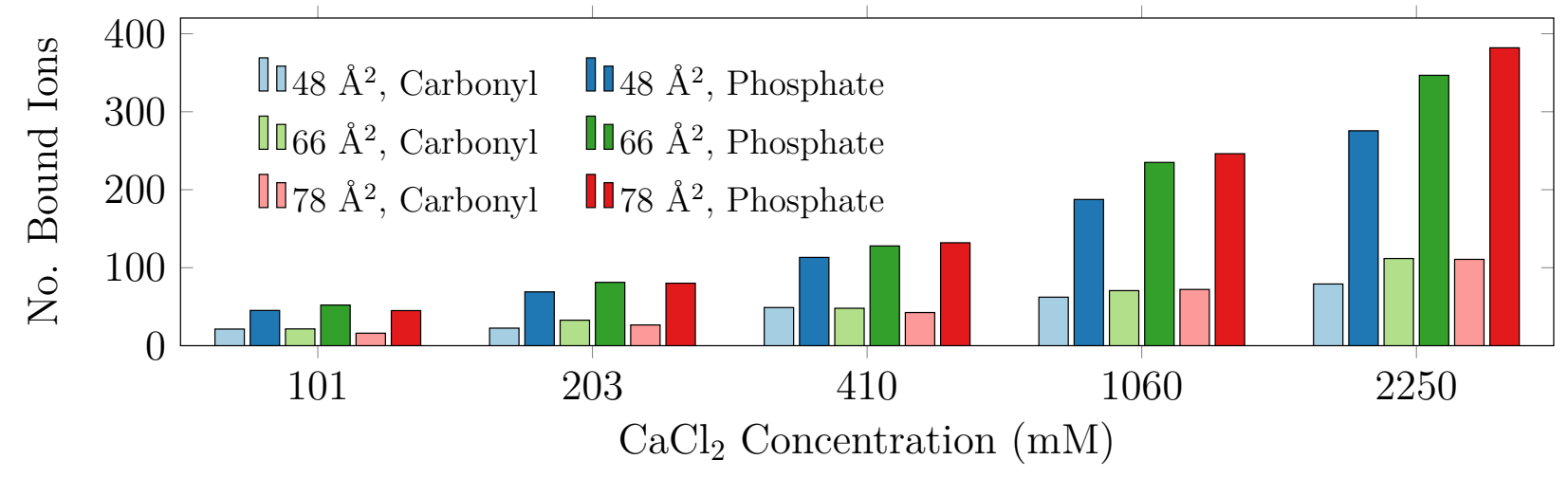

Figure 5: The binding preference of $\mathrm{Ca}^{2+}$ as a function of APL in $\AA^{2}$ (coloring) and $\mathrm{CaCl}_{2}$ concentration ( $x$-axis). The binding is resolved between carbonyl and phosphate oxygens. Notably, as a single ion can simultaneously fulfill the criteria of binding into both types of oxygens, the total number of bound ions is smaller than the sum of the two bars.

In addition to global effects manifested in phase behavior, lipid-ion interaction can also lead to conformational changes in the lipid head group. This is well described by the deuterium order parameters $S_{\mathrm{CD}}$ of the hydrocarbon segments of the lipid head group. ${ }^{8,43} \mathrm{We}$ calculated the deuterium order parameters for three carbon atoms labeled in Fig. 10 as a function of $\mathrm{CaCl}_{2}$ concentration, and the results are shown in Fig. 6 . 

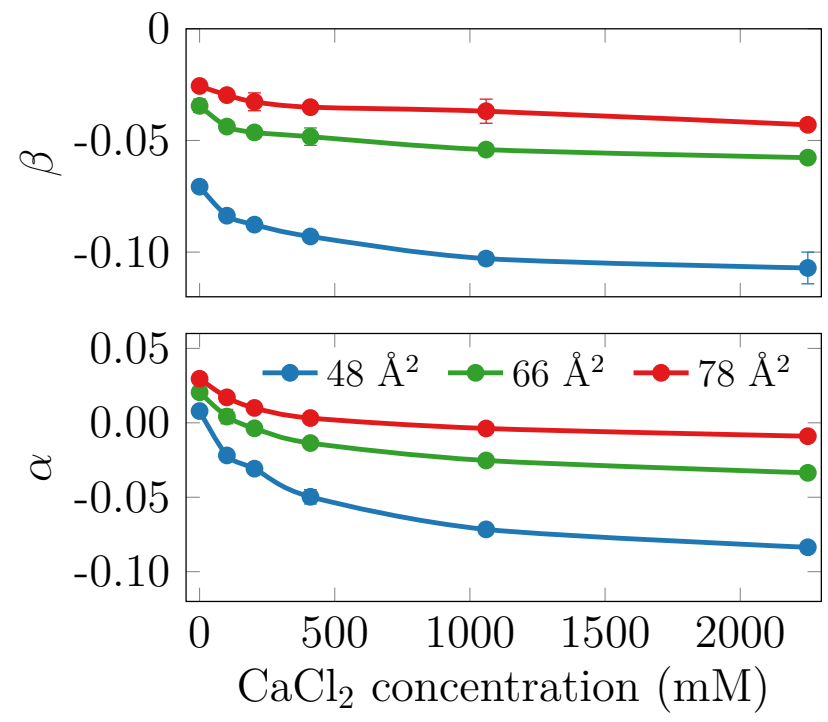

Figure 6: The dependency of lipid head group deuterium order parameters on $\mathrm{CaCl}_{2}$ concentration.

Based on Fig. 6, already a presence of a small amount of $\mathrm{CaCl}_{2}$ has a significant effect on the head group conformation. While an increasing $\mathrm{CaCl}_{2}$ concentration has an ever smaller effect on the order parameters, the change is still measureable and systematic. The shapes of the curves in Fig. 6 agree with those measured for lipid bilayers in both experiments ${ }^{43}$ and simulations with the ECC lipid model. ${ }^{13}$ Curiously, the magnitude of the effect of $\mathrm{CaCl}_{2}$ depends on monolayer packing; the smaller the monolayer APL, the more drastic the conformational changes seem to be. However, such comparison should be avoided as the dependence of the deuterium order parameter on lipid head group tilt is not linear.

To have a more detailed view on the effect of $\mathrm{CaCl}_{2}$ concentration and APL on the head group conformations, we performed clustering analysis on the lipid head group conformations. An equal number of lipid molecules was randomly selected from 18 simulations with varying area per lipid $\left(48,66\right.$, and $\left.78 \AA^{2}\right)$ and the $\mathrm{Ca}^{2+}$ concentration $(0,101,203,410$, 1060, and $2250 \mathrm{mM}$ ). According to their conformation the lipid molecules were separated into "clusters" (see Methods for details). The relative abundance of lipids from different simulations in individual clusters can be used to analyse the effect of the varying conditions (the 
area per lipid and the $\mathrm{CaCl}_{2}$ concentration) on the lipid conformation. Note that clustering algorithm is ignorant about all properties of the simulations and considers all lipid molecules as equivalent.

Since the number of conformations is not known a priori, clustering analysis was performed for different number of clusters. The conformations are distributed continuously (homogeneously covering part of the physical space) signifying that the number of clusters can not be guessed from the clustering results. Conversely, different number of clusters can be used depending on the analysis specifics - a small number of clusters (12) was used for detailed analysis of each representative conformation while a large number (64) of clusters was used especially for visual analysis of the bulk properties.

Twelve different conformations can be readily distinguished in Fig. S5 in the SI. All such clusters are significant in terms of abundance (Fig. S6 in the SI). The relative cluster abundances within each of the different simulations were analysed statistically. It turns out, that both the $\mathrm{CaCl}_{2}$ concentration and the area per lipid influences the distribution of the lipid among representative conformations (Fig. S6 in the SI). The degree (magnitude) of influence can be defined as the normalized slope of the cluster abundance as a function of the "condition" $\xi$, where $\xi$ stands either for the $\mathrm{CaCl}_{2}$ concentration or the area per lipid). Positive values represent affinity towards a high condition $\xi$, while negative values represent affinity to a low $\xi$. The affinity can also be viewed as increased probability to find a lipid of selected conformation as the condition $\xi$ increases. The magnitude of the influence was estimated through linear fitting. This is well justified as the cluster abundances clearly exhibit linear behavior with $\xi$ (Fig. S6 in the SI).

The $\mathrm{CaCl}_{2}$ concentration has a higher impact on distribution of the lipid population among the clusters than the area per lipid. A clear anti-correlation can be observed between the two in Fig. 7. In other words, if the relative abundance of a given conformation increases with $\mathrm{CaCl}_{2}$ concentration, it decreases with APL and vice versa.

Next, we focused on the effect of $\mathrm{CaCl}_{2}$ concentration on the cluster populations, as 

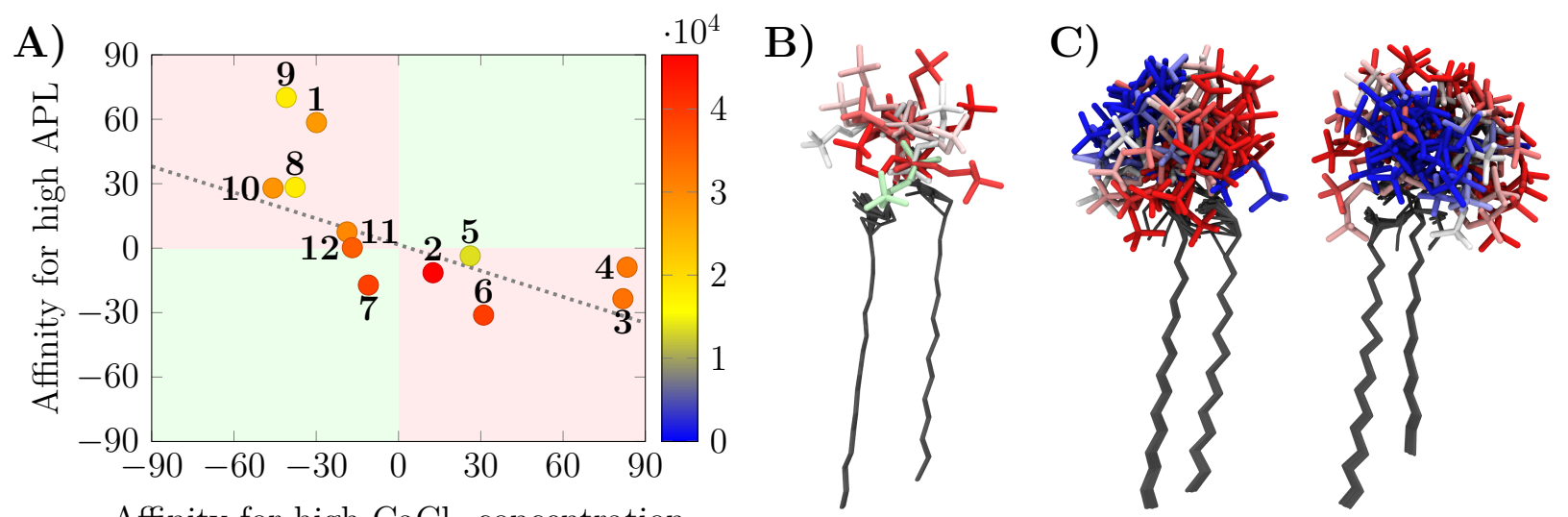

Figure 7: A) Correlation between the impacts of $\mathrm{CaCl}_{2}$ concentration and APL on the lipid head group conformation. Numbered dots signify individual clusters, where the color symbolizes the total cluster abundance. A clear anti-correlation can be observed, the conformation No. 7 being the only outlier. Linear fit of the data weighted by the relative cluster abundance (dashed gray line) exhibits a slope of -0.4 and an offset of 1.6. The range of the afinity towards $\mathrm{CaCl}_{2}$ concentration is slightly higher than for APL (-50 to +80 vs. -30 to +70$)$ signifying that the lipid conformation is affected more by the former. B) Representative conformations coloured by the correlation of the cluster abundance between the $\mathrm{CaCl}_{2}$ concentration and the APL within the respective cluster, (green: correlated, white: no correlation, red: anticorelated), the shade specifies the total $\mathrm{CaCl}_{2}$ concentration and APL dependence magnitude (light: low dependance, dark: high dependence). See http://doi.org/10.6084/m9.figshare.12783584 for additional visualization. C) The clusters - or the representative lipid conformations - can be classified by their affinity to concentrated $\mathrm{Ca}^{2+}$ environment. The measure of such affinity can be defined as a slope of the relative abundance as a function of concentration. Positive values (blue) signifies affinity to high $\mathrm{CaCl}_{2}$ concentrations while negative (red) to low ones. See http://doi.org/10.6084/m9.figshare.12783584 for additional visualization.

the effects of APL are mostly the opposite based on the anti-correlation in Fig. S6. The degree of influence can be used to further separate the clusters into two groups - those with a positive or a negative affinity towards higher $\mathrm{CaCl}_{2}$ concentrations. It turns out that the clusters are not randomly distributed amongst the groups, but rather the groups divide the physical conformation space into two areas - hemispheres (Fig. 7C). That means that the conformations with an affinity towards higher $\mathrm{CaCl}_{2}$ concentrations are physically similar. It is also evident from Fig. $7 \mathrm{C}$ that an increase in $\mathrm{CaCl}_{2}$ concentration results in more compact packing of the head group. 
To further clarify the effect of $\mathrm{CaCl}_{2}$ on the individual lipid head groups, we performed vibrational sum frequency generation (VSFG) spectroscopy measurements of the phosphate group. As shown in Fig. 8, VSFG spectroscopy reveals a substantial effect of high $\mathrm{CaCl}_{2}$ concentrations on the phosphate spectrum. The spectra at different concentrations in Fig. 8, show that the phosphate stretching peak shifts upon an increase in $\mathrm{CaCl}_{2}$ concentration. This effect likely result is in the lowered hydration of the phosphate environment due to increased presence of ions. However, at $\sim 2 \mathrm{M}$ there is an abrupt jump of the peak position in the spectra to $\sim 1143 \mathrm{~cm}^{-1}$. Moreover, the peak position saturates and a further increase in $\mathrm{CaCl}_{2}$ concentration to $2.7 \mathrm{M}$ has no visible effect on the shape nor the position of the peak. The addition of $600 \mathrm{mM}$ of $\mathrm{NaCl}$ had little effect on the spectra with the peak location aligned with that measured in the absence of salt at $\sim 1108 \mathrm{~cm}^{-1}$.

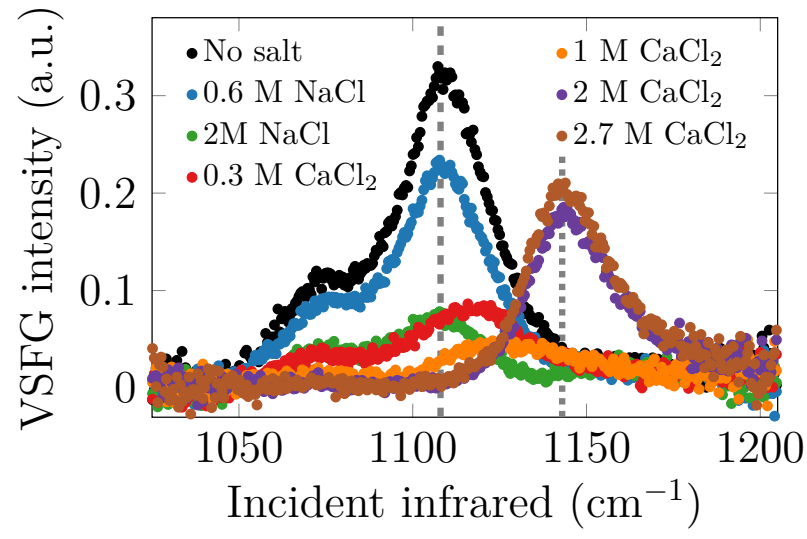

Figure 8: Vibrational sum frequency generation spectra ( $S S P$ polarization) of DPPC monolayers spread on $\mathrm{CaCl}_{2}$ subphases revealing dramatic changes in intensity and frequency coinciding with increasing calcium chloride concentrations indicative of differing binding motifs. The dashed gray line highlights the alignment of the peaks measured in the absence of salt and with $600 \mathrm{mM}$ of $\mathrm{NaCl}$ at $\sim 1108 \mathrm{~cm}^{-1}$, whereas the dotted gray line shows the alignment of the peaks at $\sim 1143 \mathrm{~cm}^{-1}$ measured for $\mathrm{CaCl}_{2}$ concentrations of 2 and $2.7 \mathrm{M}$.

To find a molecular explanation for the behavior of the VSFG spectra, we calculated the density profiles of $\mathrm{Ca}^{2+}$ and $\mathrm{Cl}^{-}$ions together with the phosphorus atoms from simulations at $66 \AA^{2}$ and at different ion concentrations. Selected profiles are shown in the top three panels of Fig. 9. Curiously, we find that the shift and saturation of the peak in the VSFG 
spectrum coincides with the penetration of $\mathrm{Cl}^{-}$ions to the region below the phosphorus atoms of DPPC. The numbers of ions that penetrate below the phosphorus density peak are plotted in the bottom panel of Fig. 9. At low concentrations, there are more $\mathrm{Ca}^{2+}$ ions in this region, yet the numbers are fairly small. However, after $\sim 700 \mathrm{mM}$, there are more $\mathrm{Cl}^{-}$ than $\mathrm{Ca}^{2+}$ ions below the phosphorus level, and at the largest studied concentration $\mathrm{Cl}^{-}$ dominates by already $\sim 45 \%$. This effect is also visually evident in Fig. 10, which shows a

typical arrangement of ions at the surface of a monolayer with an APL of $66 \AA^{2}$ at $2.25 \mathrm{M}$ of $\mathrm{CaCl}_{2}$. The $\mathrm{Ca}^{2+}$ ions, shown in yellow, are bound to the phosphate group, whereas the $\mathrm{Cl}^{-}$ions, shown in green, can penetrate to beyond this region and all the way to the vicinity of the carbonyl groups. Since the carbonyl oxygens bear a negative partial charge, this arrangement is likely stabilized by the overcharging at the phosphate level caused by the large amounts of $\mathrm{Ca}^{2+}$ ions therein. ${ }^{44}$

\section{Discussion and Conclusions}

In this work, we combined molecular dynamics simulations with Langmuir trough measurements and vibrational sum frequency generation spectroscopy to unravel the effect of cations on the properties of lipid monolayers. To this end, we have simulated DPPC monolayers at various compression states and in the presence of $\mathrm{NaCl}$ and $\mathrm{CaCl}_{2}$. The force fields used for DPPC, water, and ions were all state-of-the-art and accurately describe the behavior of lipids at water-air interfaces ${ }^{19}$ as well as the lipid-ion interactions. ${ }^{13}$ The simulated systems cover all monolayer phases - liquid condensed and liquid expanded phases and their coexistence. For $\mathrm{CaCl}_{2}$, additional simulations where performed with multiple salt concentrations for three compression states that correspond to the two studied monolayer phases and their coexistence. Experimentally, we evaluated the effects of $\mathrm{NaCl}$ and $\mathrm{CaCl}_{2}$ on Langmuir isotherms and studied the effect of various $\mathrm{CaCl}_{2}$ concentrations on the vibrational spectrum of the phosphate group in the DPPC head group. 

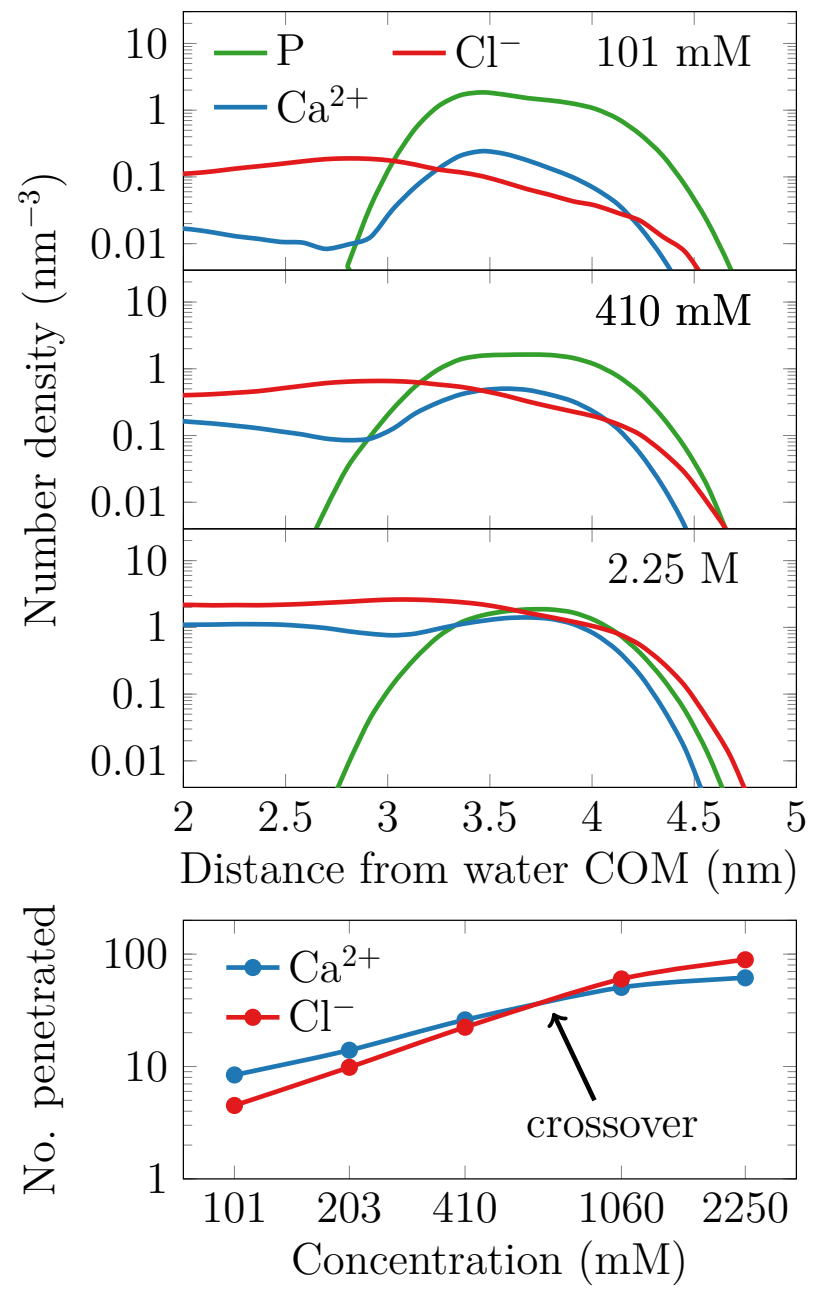

Figure 9: Top three panels: Number density profiles of phosphorus (P), calcium and chloride on the monolayer surface at an APL of $66 \AA^{2}$ as a function of $\mathrm{CaCl}_{2}$ concentration for selected systems. Bottom panel: The number of ions that have penetrated below the phosphorus level, shown in the $\log -\log$ scale. At $\sim 700 \mathrm{mM}$, the number of penetrated $\mathrm{Cl}^{-}$becomes larger than that of $\mathrm{Ca}^{2+}$.

We first obtained surface pressure-area isotherms from both experiments and simulations. From these isotherms in Fig. 2, it is evident that the shapes of the simulated and experimental isotherms are not identical. Nevertheless, the simulated isotherm reproduces the characteristic plateau of DPPC isotherms ${ }^{19}$ and the surface pressure values are mostly reasonable without any shifting of the isotherms. ${ }^{12}$ Moreover, it seems that at small areas per lipid the current ECC-based model performs better than CHARMM36 in our earlier work, ${ }^{19}$ whereas at larger areas CHARMM36 is in better agreement with experiment. Strikingly, at 


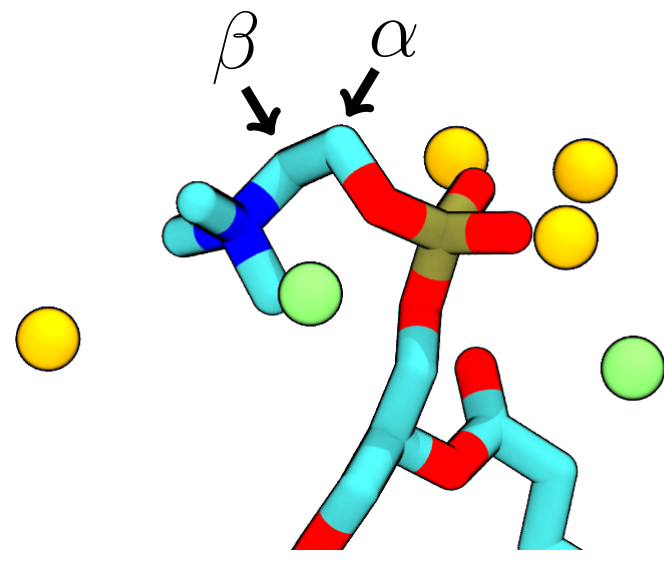

Figure 10: Characteristic conformation of the DPPC head group and bound ions under high $\mathrm{CaCl}_{2}$ concentrations. Here, carbons are shown in cyan, oxygen in red, phosphorus in brown, and nitrogen in blue. $\mathrm{Ca}^{2+}$ and $\mathrm{Cl}^{-}$ions are shown in yellow and green, respectively. Hydrogens and water are omitted for clarity. The labels indicate the carbons for which deuterium order parameters are reported in Fig. 6.

APL equal to $54 \AA^{2}$, the surface pressure in the system with $\mathrm{CaCl}_{2}$ reaches a negative value. It seems that at this area, $\mathrm{CaCl}_{2}$ is able to cluster the lipids efficiently, which in a finite simulation box leads to a phase separation between tightly-packed clusters and small amounts of exposed surface. Despite these limitations, the simulation model seems to capture well the effect of ions on the pressure-area isotherms and justifies our attempts to explain the experimentally observed behavior based on simulation data.

We then evaluated how monolayer packing and cation type affect the adsorption of ions to the monolayer surface. The dependence of cation-lipid contacts on APL (bottom panel in Fig. 2) agrees well with our recent study ${ }^{6}$ despite the fact that no ECC lipids were used therein: $\mathrm{Na}^{+}$binding depends only on the amount of PC head groups available for binding, whereas the adsorption of $\mathrm{Ca}^{2+}$ depends on the surface area available for binding. The saturation of $\mathrm{Ca}^{2+}$ binding at large areas (bottom panel in Fig. 2) is a new observation, and likely results from the fact that the loosely-packed lipid-water interface undergoes charge inversion. This is more likely with the ECC lipids used in this work (in contrast to Ref. 6), as the inversion happens at a smaller amount of bound $\mathrm{Ca}^{2+}$.

Both simulations and experiments demonstrated a condensing effect of $\mathrm{CaCl}_{2}$ on the 
monolayers, whereas $\mathrm{NaCl}$ was not found to have an effect on lipid packing. The effect of $\mathrm{CaCl}_{2}$ was most present in the $\mathrm{L}_{\mathrm{e}} / \mathrm{L}_{\mathrm{c}}$ coexistence region, where the surface pressures were lowered by $\mathrm{CaCl}_{2}$. The simulations revealed that this condensation resulted from an increase in the fraction of the $\mathrm{L}_{\mathrm{c}}$ phase, whereas the properties of the individual phases were not affected. Simulations with varying $\mathrm{CaCl}_{2}$ concentrations demonstrated that the condensing effect of $\mathrm{CaCl}_{2}$ is strong at relatively small concentrations and the effects saturate at $\sim 400 \mathrm{mM}$. Still, a further increase in $\mathrm{CaCl}_{2}$ concentration affected the conformations of lipid head groups (Figs. 6 and 7). Our cluster analysis revealed that increases in $\mathrm{CaCl}_{2}$ concentration and APL affect the cluster populations in an anti-correlated way. Moreover, we observed that the clusters that are more preferred at higher and lower $\mathrm{CaCl}_{2}$ concentrations are physically distinct and populate two hemispheres. Moreover, higher $\mathrm{CaCl}_{2}$ concentrations also prefer more compact head group conformations (Fig. 7). At concentrations above $2 \mathrm{M}$, the VSFG spectra (Fig. 8) displayed drastic effects in the phosphate region. Unfortunately, due to its quantum-mechanical nature, a VSFG spectrum cannot be resolved from our atomistic simulations. Moreover, performing quantum-mechanical calculations for all the lipid-cation geometries observed in our simulations is out of the reach of current resources. Therefore, we connected the VSFG spectra to properties available in our simulations, namely ion densities and binding modes. This approach revealed (Fig. 5) that at high $\mathrm{CaCl}_{2}$ concentrations the binding to the carbonyl groups is fairly limited, whereas more ions adsorbed to the phosphate region and this binding was coupled with $\mathrm{Cl}^{-}$ions penetrating deep below the phosphate region (Fig. 9). This arrangement with $\mathrm{Cl}^{-}$ions allowing more $\mathrm{Ca}^{2+}$ to bind to the phosphate region appears only at high $\mathrm{CaCl}_{2}$ concentrations. We believe that this specific organization of ions is the new binding mode that appears in the VSFG spectrum at high $\mathrm{CaCl}_{2}$ concentrations.

Based on our results, the changes in lipid packing take place at fairly low $\mathrm{CaCl}_{2}$ concentrations and especially in the $\mathrm{L}_{\mathrm{c}} / \mathrm{L}_{\mathrm{e}}$ coexistence region. However, lipid head group conformations are systematically affected by an increasing $\mathrm{CaCl}_{2}$ concentration at least until the largest 
simulated concentration of $2.25 \mathrm{M}$. Curiously, the head group conformations of the $\mathrm{L}_{\mathrm{c}}$ phase monolayer are most affected by $\mathrm{CaCl}_{2}$. Finally, very large $\mathrm{CaCl}_{2}$ concentrations introduce a new and mutual binding mode for $\mathrm{Ca}^{2+}$ and $\mathrm{Cl}^{-}$detected in VSFG spectroscopy and our density profiles. Thus it seems that the local (conformation and ion binding mode of a single lipid) and global (lateral packing of lipids) effects are not connected in a straightforward manner.

One limitation of the ECC framework worth mentioning here is that it is in principle limited to studies on systems that are heterogeneous in their electronic response. This is obviously not true for interfaces where the electronic polarizabilty changes abruptly, and different scaling factors should therefore be applied on the two sides of the interface, 1 for the air phase and 0.75 for the aqueous phase. ${ }^{26}$ We are not aware of any simulation code which allows the definition of position-dependent ECC scaling factors or dielectric constants. However, this should be possible to implement, as the profile of the dielectric constant at the interface is known. ${ }^{45}$ Here, the use of the constant scaling factor of 0.75 leads to a too weak effect of salt on surface tension of the electrolyte solution, whereas the effect with unscaled charges is overestimated. ${ }^{26}$ Still, we consider this limitation acceptable, as the use of ECC ions remedies the more severe issue of ion-lipid overbinding.

Our results help us understand how ions can regulate local lipid packing in the DPPC-rich pulmonary surfactant monolayers, where the phase behavior of lipids is of key physiological importance. Namely, the balance of $\mathrm{L}_{\mathrm{e}}$ and $\mathrm{L}_{\mathrm{c}}$ phases is required for the proper folding of the pulmonary surfactant monolayer into lipid reservoirs during exhaling, as well as for the correct function of surfactant proteins. ${ }^{22}$ However, application of our results to cellular membranes is likely less straightforward, as the behavior of monolayers used here depend heavily on their compression state. Still, the trends observed here especially for the head group conformations are likely similar despite the level of lipid packing. Still, cellular membranes are very complex entities with thousands of lipid components, proteins, and other molecules. Notably, cellular membranes have other means to control lipid packing, the most well-known 
one being sterol molecules such as cholesterol. ${ }^{46}$ It is also worth noting that while the concentrations used here for $\mathrm{NaCl}$ are close to those present in the extracellular fluid (145 mM), the used $\mathrm{CaCl}_{2}$ concentrations are drastically higher than those present therein $(1-2 \mathrm{mM}) .{ }^{47}$ In the cytosol, $\mathrm{CaCl}_{2}$ is much more scarce, and its concentration remains smaller than in the extracellular fluid even during signaling-related calcium spikes. ${ }^{48}$ Therefore, the possible applications of the high $\mathrm{Ca}_{2+}$ concentrations used in this study likely lie in the field of engineering and in marine-derived atmospheric aerosol chemistry, rather than in biosciences.

\section{Acknowledgement}

M.J. thanks CSC-IT Center for Science for computational resources and the Emil Aaltonen foundation for funding. M.J. and O.T. acknowledge computational resources supplied by the project "e-Infrastruktura CZ" (e-INFRA LM2018140) provided within the program Projects of Large Research, Development and Innovations Infrastructures. M.J. and L.C. acknowledge the support from the Czech Science Foundation (EXPRO Grant 19-26854X). W.H. acknowledges Mr. Zishuai Huang for his assistance in the preparation of sample solutions. H.C.A. and W.H. acknowledge the support from the U. S. National Science Foundation (NSF) grant CHE-1111762, and through the NSF Center for Aerosol Impacts on Chemistry on the Environment, grant CHE-1305427). O.T. acknowledges the Faculty of Mathematics and Physics of the Charles University (Prague, Czech Republic) where he is enrolled as a PhD student and the International Max Planck Research School for "Many-Particle Systems in Structured Environments" (Dresden, Germany) for support. P.D. acknowledges the support from the

Czech Science Foundation (grant 17-06792S). We thank Dr. Samuli Ollila for constructive comments.

\section{Supporting Information Available}

Optimization of the ECC ions for OPC water. Computational results on the surface tension 
of the lipid-free interface, on the convergence of the ion adsorption, on the $\mathrm{Ca}^{2+}$ binding preference as a function of area per lipid, and additional results on the clustering of lipid head group structures.

\section{References}

(1) Dodd, A. N.; Kudla, J.; Sanders, D. The Language of Calcium Signaling. Ann. Rev. Plant. Biol. 2010, 61, 593-620.

(2) Verdaguer, N.; Corbalan-Garcia, S.; Ochoa, W. F.; Fita, I.; Gómez-Fernández, J. C. $\mathrm{Ca}^{2+}$ Bridges the C2 Membrane-Binding Domain of Protein Kinase $\mathrm{C} \alpha$ Directly to Phosphatidylserine. EMBO J. 1999, 18, 6329-6338.

(3) Bilkova, E.; Pleskot, R.; Rissanen, S.; Sun, S.; Czogalla, A.; Cwiklik, L.; Róg, T.; Vattulainen, I.; Cremer, P. S.; Jungwirth, P.; Coskun, U. Calcium Directly Regulates Phosphatidylinositol 4,5-Bisphosphate Headgroup Conformation and Recognition. J. Am. Chem. Soc. 2017, 139, 4019-4024.

(4) Boettcher, J. M.; Davis-Harrison, R. L.; Clay, M. C.; Nieuwkoop, A. J.; Ohkubo, Y. Z.; Tajkhorshid, E.; Morrissey, J. H.; Rienstra, C. M. Atomic View of Calcium-Induced Clustering of Phosphatidylserine in Mixed Lipid Bilayers. Biochemistry 2011, 50, 22642273.

(5) Sarmento, M. J.; Coutinho, A.; Fedorov, A.; Prieto, M.; Fernandes, F. Ca ${ }^{2+}$ Induces $\mathrm{PI}(4,5) \mathrm{P}_{2}$ Clusters on Lipid Bilayers at Physiological $\mathrm{PI}(4,5) \mathrm{P}_{2}$ and $\mathrm{Ca}^{2+}$ Concentrations. BBA-Biomembranes 2014, 1838, 822-830.

(6) Javanainen, M.; Melcrová, A.; Magarkar, A.; Jurkiewicz, P.; Hof, M.; Jungwirth, P.; Martinez-Seara, H. Two Cations, Two Mechanisms: Interactions of Sodium and Calcium With Zwitterionic Lipid Membranes. Chem. Comm. 2017, 53, 5380-5383. 
(7) Melcrová, A.; Pokorna, S.; Pullanchery, S.; Kohagen, M.; Jurkiewicz, P.; Hof, M.; Jungwirth, P.; Cremer, P. S.; Cwiklik, L. The Complex Nature of Calcium Cation Interactions With Phospholipid Bilayers. Sci. Rep. 2016, 6, 38035.

(8) Catte, A.; Girych, M.; Javanainen, M.; Loison, C.; Melcr, J.; Miettinen, M.; Monticelli, L.; Määttä, J.; Oganesyan, V.; Ollila, O.; Tynkkynen, J.; Vilov, S. The Electrometer Concept and Binding of Cations to Phospholipid Bilayers. Phys. Chem. Chem. Phys. 2016,

(9) Böckmann, R. A.; Grubmüller, H. Multistep Binding of Divalent Cations to Phospholipid Bilayers: A Molecular Dynamics Study. Angew. Chem. Int. Ed. 2004, 43, $1021-1024$.

(10) Ishiyama, T.; Shirai, S.; Okumura, T.; Morita, A. Molecular Dynamics Study of Structure and Vibrational Spectra at Zwitterionoic Lipid/Aqueous $\mathrm{KCl}, \mathrm{NaCl}$, and $\mathrm{CaCl}_{2}$ Solution Interfaces. J. Chem. Phys. 2018, 148, 222801.

(11) Simons, K.; Vaz, W. L. Model Systems, Lipid Rafts, and Cell Membranes. Annu. Rev. Biophys. Biomol. Struct. 2004, 33, 269-295.

(12) Lamberg, A.; Ollila, O. S. Comment on "Structural Properties of POPC Monolayers Under Lateral Compression: Computer Simulations Analysis". Langmuir 2015, 31, 886-887.

(13) Melcr, J.; Martinez-Seara, H.; Nencini, R.; Kolafa, J.; Jungwirth, P.; Ollila, O. S. Accurate Binding of Sodium and Calcium to a POPC Bilayer by Effective Inclusion of Electronic Polarization. J. Phys. Chem. B 2018, 122, 4546-4557.

(14) Kohagen, M.; Mason, P. E.; Jungwirth, P. Accounting for Electronic Polarization Effects in Aqueous Sodium Chloride via Molecular Dynamics Aided by Neutron Scattering. J. Phys. Chem. B 2015, 120, 1454-1460. 
(15) Martinek, T.; Duboué-Dijon, E.; Timr, Š.; Mason, P. E.; Baxová, K.; Fischer, H. E.; Schmidt, B.; Pluhařová, E.; Jungwirth, P. Calcium Ions in Aqueous Solutions: Accurate Force Field Description Aided by ab initio Molecular Dynamics and Neutron Scattering. J. Chem. Phys. 2018, 148, 222813.

(16) Pluhařová, E.; Fischer, H. E.; Mason, P. E.; Jungwirth, P. Hydration of the Chloride Ion in Concentrated Aqueous Solutions Using Neutron Scattering and Molecular Dynamics. Mol. Phys. 2014, 112, 1230-1240.

(17) Dickson, C. J.; Madej, B. D.; Skjevik, A. A.; Betz, R. M.; Teigen, K.; Gould, I. R.; Walker, R. C. Lipid14: The Amber Lipid Force Field. J. Chem. Theory Comput. 2014, 10, 865-879.

(18) Izadi, S.; Anandakrishnan, R.; Onufriev, A. V. Building Water Models: A Different Approach. J. Phys. Chem. Lett. 2014, 5, 3863-3871.

(19) Javanainen, M.; Lamberg, A.; Cwiklik, L.; Vattulainen, I.; Ollila, O. S. Atomistic Model for Nearly Quantitative Simulations of Langmuir Monolayers. Langmuir 2017, $34,2565-2572$.

(20) Liekkinen, J.; de Santos Moreno, B.; Paananen, R. O.; Vattulainen, I.; Monticelli, L.; de la Serna, J. B.; Javanainen, M. Understanding the Functional Properties of Lipid Heterogeneity in Pulmonary Surfactant Monolayers at the Atomistic Level. bioRxiv 2020,

(21) Olżyńska, A.; Zubek, M.; Roeselova, M.; Korchowiec, J.; Cwiklik, L. Mixed DPPC/POPC Monolayers: All-Atom Molecular Dynamics Simulations and Langmuir Monolayer Experiments. BBA-Biomembranes 2016, 1858, 3120-3130.

(22) Pérez-Gil, J. Structure of Pulmonary Surfactant Membranes and Films: The Role of Proteins and Lipid-protein Interactions. BBA - Biomembranes 2008, 1778, 1676-1695. 
(23) Leontyev, I.; Stuchebrukhov, A. Accounting for Electronic Polarization in NonPolarizable Force Fields. Phys. Chem. Chem. Phys. 2011, 13, 2613-2626.

(24) Kirby, B. J.; Jungwirth, P. Charge Scaling Manifesto: A Way of Reconciling the Inherently Macroscopic and Microscopic Natures of Molecular Simulations. J. Phys. Chem. Lett. 2019, 10, 7531-7536.

(25) Melcr, J.; Piquemal, J.-P. Accurate Biomolecular Simulations Account for Electronic Polarization. Frontiers in Molecular Biosciences 2019, 6.

(26) DubouÃl'-Dijon, E.; Javanainen, M.; Delcroix, P.; Jungwirth, P.; Martinez-Seara, H. A Practical Guide to Biologically-Relevant Molecular Simulations With Charge Scaling for Electronic Polarization. J. Chem. Phys. 2020, 153, 050901.

(27) Abraham, M. J.; Murtola, T.; Schulz, R.; Páll, S.; Smith, J. C.; Hess, B.; Lindahl, E. GROMACS: High Performance Molecular Simulations Through Multi-level Parallelism From Laptops to Supercomputers. SoftwareX 2015, 1, 19-25.

(28) Essman, U.; Perera, L.; Berkowitz, M.; Darden, T.; Lee, H.; Pedersen, L. A Smooth Particle Mesh Ewald Potential. J. Chem. Phys 1995, 103, 8577-8592.

(29) Shirts, M. R.; Mobley, D. L.; Chodera, J. D.; Pande, V. S. Accurate and Efficient Corrections for Missing Dispersion Interactions in Molecular Simulations. J. Phys. Chem. B 2007, 111, 13052-13063.

(30) Páll, S.; Hess, B. A Flexible Algorithm for Calculating Pair Interactions on SIMD Architectures. Comput. Phys. Commun. 2013, 184, 2641-2650.

(31) Bussi, G.; Donadio, D.; Parrinello, M. Canonical Sampling Through Velocity Rescaling. J. Chem. Phys. 2007, 126, 014101.

(32) Hess, B.; Bekker, H.; Berendsen, H. J.; Fraaije, J. G. LINCS: A Linear Constraint Solver for Molecular Simulations. J. Comput. Chem. 1997, 18, 1463-1472. 
(33) Hess, B. P-LINCS: A Parallel Linear Constraint Solver for Molecular Simulation. J. Chem. Theory Comput. 2008, 4, 116-122.

(34) Michaud-Agrawal, N.; Denning, E. J.; Woolf, T. B.; Beckstein, O. MDAnalysis: A Toolkit for the Analysis of Molecular Dynamics Simulations. J. Comput. Chem. 2011, 32, 2319-2327.

(35) McAdams, A.; Selle, A.; Tamstorf, R.; Teran, J.; Sifakis, E. Computing the Singular Value Decomposition of 3x3 Matrices With Minimal Branching and Elementary Floating Point Operations; 2011.

(36) Humphrey, W.; Dalke, A.; Schulten, K. VMD - Visual Molecular Dynamics. J. Mol. Graph. 1996, 14, 33-38.

(37) Stone, J. An Efficient Library for Parallel Ray Tracing and Animation. M.Sc. thesis, Computer Science Department, University of Missouri-Rolla, 1998.

(38) Hua, W.; Verreault, D.; Adams, E. M.; Huang, Z.; Allen, H. C. Impact of Salt Purity on Interfacial Water Organization Revealed by Conventional and Heterodyne-Detected Vibrational Sum Frequency Generation Spectroscopy. J. Phys. Chem. C 2013, 117, $19577-19585$.

(39) Finlayson, A. The pH range of the Mohr Titration for Chloride Ion Can Be Usefully Extended to 4-10.5. J. Chem. Educ. 1992, 69, 559.

(40) Hua, W.; Verreault, D.; Huang, Z.; Adams, E. M.; Allen, H. C. Cation Effects on Interfacial Water Organization of Aqueous Chloride Solutions. I. Monovalent Cations: $\mathrm{Li}^{+}, \mathrm{Na}^{+}, \mathrm{K}^{+}$, and $\mathrm{NH}_{4}^{+}$. J. Phys. Chem. B 2014, 118, 8433-8440.

(41) Adams, E. M.; Casper, C. B.; Allen, H. C. Effect of Cation Enrichment on Dipalmitoylphosphatidylcholine (DPPC) Monolayers at the Air-Water Interface. J. Colloid Interface Sci. 2016, 478, 353-364. 
(42) Dean, J. A. Lange's Handbook of Chemistry; New york; London: McGraw-Hill, Inc., 1999.

(43) Akutsu, H.; Seelig, J. Interaction of Metal Ions With Phosphatidylcholine Bilayer Membranes. Biochemistry 1981, 20, 7366-7373.

(44) Hua, W.; Verreault, D.; Allen, H. C. Solvation of Calcium-Phosphate Headgroup Complexes at the DPPC/Aqueous Interface. ChemPhysChem 2015, 16, 3910-3915.

(45) Shiratori, K.; Morita, A. Molecular Theory on Dielectric Constant at Interfaces: A Molecular Dynamics Study of the Water/Vapor Interface. J. Chem. Phys. 2011, 134, 234705 .

(46) Róg, T.; Pasenkiewicz-Gierula, M.; Vattulainen, I.; Karttunen, M. Ordering Effects of Cholesterol and Its Analogues. BBA - Biomembranes 2009, 1788, 97-121.

(47) Alberts, B.; Bray, D.; Hopkin, K.; Johnson, A. D.; Lewis, J.; Raff, M.; Roberts, K.; Walter, P. Essential Cell Biology; Garland Science, 2013.

(48) Ross, W. N. Understanding Calcium Waves and Sparks in Central Neurons. Nat. Rev. Neurosci. 2012, 13, 157. 


\section{Graphical TOC Entry}

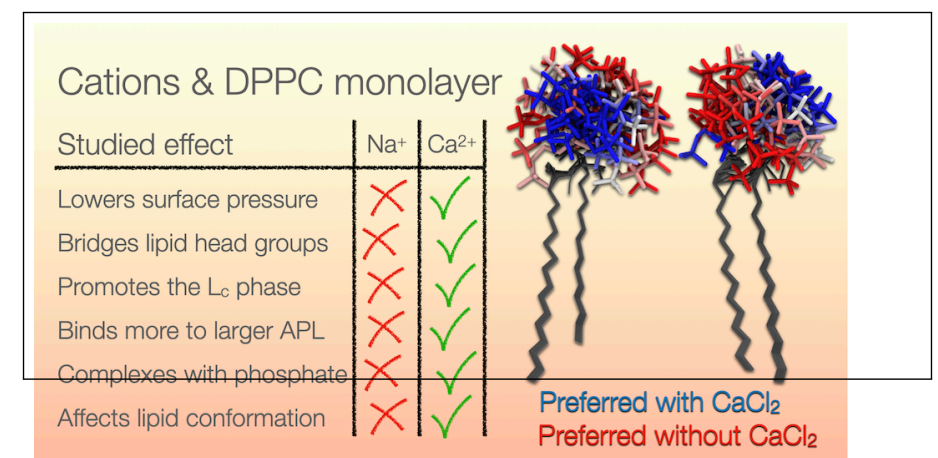

